\title{
Cutaneous angiosarcoma after breast conserving treatment of breast cancer
}

\author{
W. Eiermann, B. Ataseven \\ Frauenklinik vom Roten Kreuz, Munich, Germany.
}

\section{Case report}

In this case we describe a 77-year-old female who underwent breast conserving therapy, adjuvant radiotherapy and endocrine therapy (tamoxifen) for primary breast cancer (pT2, pN0, G3, R0, Mx, hormone receptor positive, Her2-negative). 28 month after completion of adjuvant radiotherapy new purple plaques and discolouration in the radiotherapy field were seen on the left breast, without other signs for example, lymphedema, inflammation, pain or itch. The histological result of a biopsy revealed a atypical vascular process (differential diagnosis: well differentiated

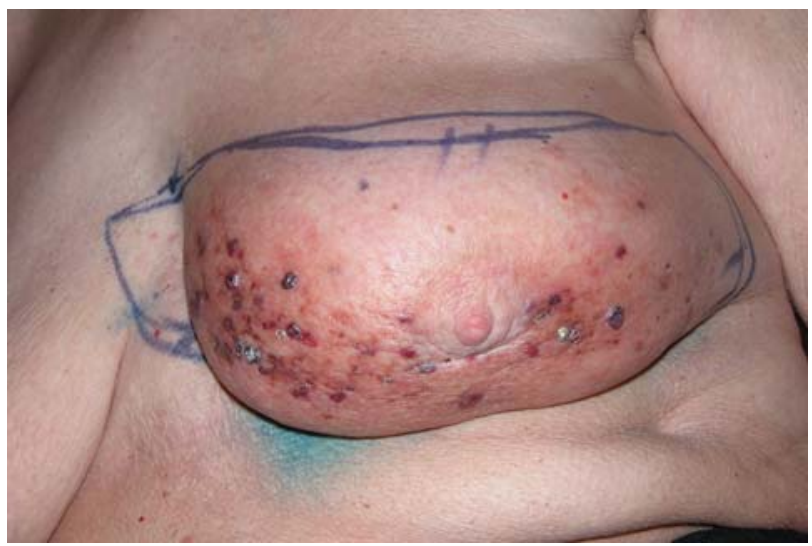

(a)

Figure 1.

Left breast with multiple purple, red and red-brown papulonodular skin lesions up to $1.3 \mathrm{~cm}$. Retraction of the nipple with in duration behind.

Correspondence to: B. Ataseven, Frauenklinik vom Roten Kreuz, Munich Germany. E-mail: beyhan.ataseven@swmbrk.de Tel: +49 89 15706620; Fax: +498915706623

Received: 07/01/05

Accepted: 26/04/05

First published online 25/09/06

BCO/410/2005/CS angiosarcoma), no further differentiation was possible. At first a temporary improvement was seen. But 5 month later a clear progress happened. Now multifocal purple and red-brown coloured lesions up to $1.3 \mathrm{~cm}$ were spread all over the left breast (Fig. 1(a) and (b)). A second biopsy confirmed histological the

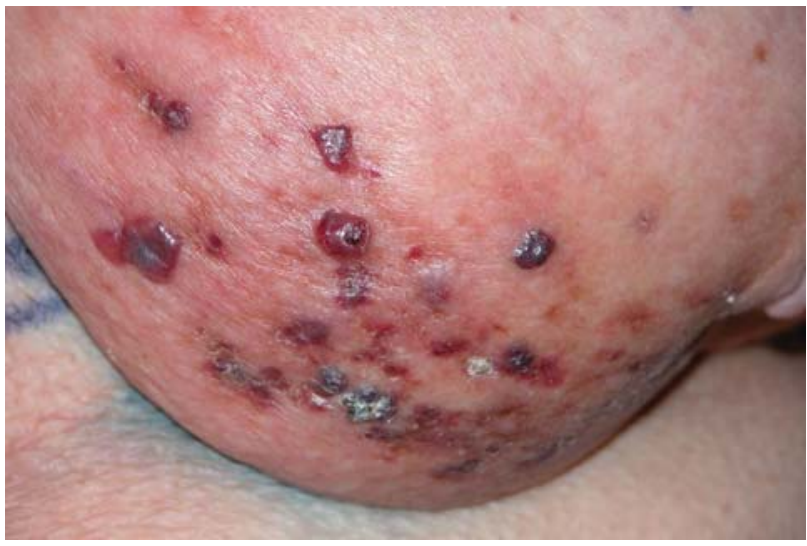

(b) (Continued)

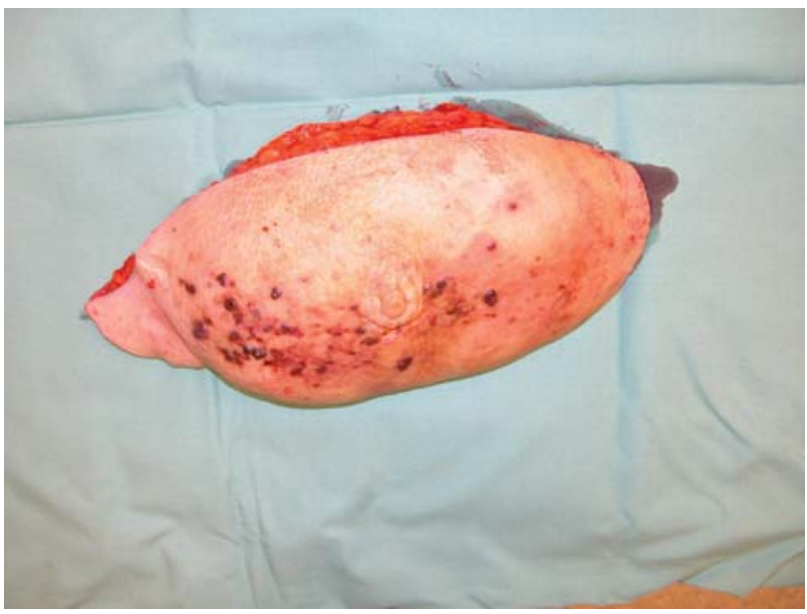

(c) (Continued) 
diagnosis of cutaneous angiosarcoma (well differentiated). There were no sign for intraglandular lump or suspicious axillary lymph node. The supra- and infraclavicular fossae were although free, so was the mammogram.

We performed simple mastectomy (Fig. 1(c)). All lesions could be removed with clear margins. Multiple cutaneous angiosarcoma up to $1.3 \mathrm{~cm}$ were detectable. The breast tissue lacked signs for recurrence of previously detected breast cancer. After surgery radiotherapy was applied. The treatment with tamoxifen for breast cancer which was started before continued. The patient was alive without recurrence after 12 month.

For local control after breast conserving therapy for primary breast cancer radiotherapy is an accepted part of treatment. Cutaneous angiosarcomas are rare neoplasms. Beside radiation, exposure to sunlight or chronic lymphoedema (Stewart-Treves syndrome) are important aetiological factors. In retrospective studies cutaneous angiosarcoma we seen in a frequency of 1:2000 to 1:3000 mostly many years after radiotherapy. The incidence is be expected to increase. Due to explosive growth and extensive recurrence the prognosis is poor.

Therefore increasing attention must be given to a patient with skin alteration after radiotherapy. These lesions are difficult to diagnose sometimes they mimic radiation dermatitis and atypical vascular lesions. Biopsy of these areas must be done. Early surgery is an important part of therapy. The success of chemotherapy is uncertain. For this aggressive disease doxorubicin and actinomycin $\mathrm{D}$ are rare tested agents. Convincing results were not seen, therefore chemotherapy is recommended mainly in advanced stages of disease. Further investigation of chemotherapy for cutaneous angiosarcoma should be done. Angiosarcoma is mostly sensible for hyperfractionated radiotherapy. In absense of contraindications radiotherapy is recommended. Followup is recommended in frequent intervals. Skin inspectation should be performed extremely carefully, sometimes it is helpful to document by series of photographies in order to compare and detect early changes. 